\title{
Setup presentation and clinical outcome analysis of treating highly language-eloquent gliomas via preoperative navigated transcranial magnetic stimulation and tractography
}

\author{
Nico Sollmann, MD, PhD, ${ }^{1-3}$ Anna Kelm, ${ }^{2,3}$ Sebastian IIle, MD,, ${ }^{2,3}$ Axel Schröder, ${ }^{2}$ \\ Claus Zimmer, MD, ${ }^{1,3}$ Florian Ringel, MD, ${ }^{2}$ Bernhard Meyer, MD, ${ }^{2}$ and Sandro M. Krieg, MD, MBA ${ }^{2,3}$ \\ ${ }^{1}$ Department of Diagnostic and Interventional Neuroradiology, ${ }^{2}$ Department of Neurosurgery, and ${ }^{3}$ TUM-Neuroimaging Center, \\ Klinikum rechts der Isar, Technische Universität München, Germany
}

\begin{abstract}
OBJECTIVE Awake surgery combined with intraoperative direct electrical stimulation (DES) and intraoperative neuromonitoring (IONM) is considered the gold standard for the resection of highly language-eloquent brain tumors. Different modalities, such as functional magnetic resonance imaging (fMRI) or magnetoencephalography (MEG), are commonly added as adjuncts for preoperative language mapping but have been shown to have relevant limitations. Thus, this study presents a novel multimodal setup consisting of preoperative navigated transcranial magnetic stimulation (nTMS) and nTMS-based diffusion tensor imaging fiber tracking (DTI FT) as an adjunct to awake surgery.

METHODS Sixty consecutive patients (63.3\% men, mean age $47.6 \pm 13.3$ years) suffering from highly language-eloquent left-hemispheric low- or high-grade glioma underwent preoperative nTMS language mapping and nTMS-based DTI FT, followed by awake surgery for tumor resection. Both nTMS language mapping and DTI FT data were available for resection planning and intraoperative guidance. Clinical outcome parameters, including craniotomy size, extent of resection (EOR), language deficits at different time points, Karnofsky Performance Scale (KPS) score, duration of surgery, and inpatient stay, were assessed.

RESULTS According to postoperative evaluation, $28.3 \%$ of patients showed tumor residuals, whereas new surgery-related permanent language deficits occurred in $8.3 \%$ of patients. KPS scores remained unchanged (median preoperative score 90, median follow-up score 90).

CONCLUSIONS This is the first study to present a clinical outcome analysis of this very modern approach, which is increasingly applied in neurooncological centers worldwide. Although human language function is a highly complex and dynamic cortico-subcortical network, the presented approach offers excellent functional and oncological outcomes in patients undergoing surgery of lesions affecting this network.

https://thejns.org/doi/abs/10.3171/2018.3.FOCUS1838
\end{abstract}

KEYWORDS awake surgery; brain tumor; direct electrical stimulation; language mapping; navigated transcranial magnetic stimulation

I $\mathrm{N}$ modern neurooncology, awake surgery combined with intraoperative direct electrical stimulation (DES) and intraoperative neuromonitoring (IONM) reflects the standard for resection of language-eloquent brain tumors. ${ }^{19,46,48,61,62}$ This approach is favored over resection under general anesthesia due to the fact that it allows for language mapping during surgery, which aims to detect and spatially enclose language-related cortical and subcortical structures as an efficient way to reduce postoperative language deficits. ${ }^{6.46} \mathrm{Within}$ the context of the so-called oncofunctional balance, a minimum rate of postoperative deficits should be achieved in combination with maximum

ABBREVIATIONS AP = anterior-posterior; BOLD = blood oxygenation level-dependent; DES = direct electrical stimulation; $\mathrm{DTI}=$ diffusion tensor imaging; DTI FT = DTI fiber tracking; EOR = extent of resection; $\mathrm{FMRI}$ = functional MRI; GTR = gross-total resection; HGG = high-grade glioma; IONM = intraoperative neuromonitoring; KPS = Karnofsky Performance Scale; LGG = low-grade glioma; MEG = magnetoencephalography; nTMS = navigated transcranial magnetic stimulation; rMT = resting motor threshold; STR = subtotal resection. 
tumor resection, thus ideally leading to optimal postoperative quality of life and prolonged patient survival., 2,10,36,47

However, the gold standard of awake surgery with DES and IONM can be supported by various preoperative adjuncts that allow for mapping of language function prior to tumor resection, which enables the treating neurosurgeon to assess functionally critical cortical and subcortical structures in advance. ${ }^{39}$ Among the most common options in clinical routine, functional MRI (fMRI) and magnetoencephalography (MEG) have been applied successfully. ${ }^{16,35,39,40,43,44}$ In addition, navigated transcranial magnetic stimulation (nTMS) has been introduced recently as a novel technique for preoperative language mapping in patients with brain tumors. ${ }^{42,56,63}$ By using a magnetic coil, magnetic pulses can be delivered over preselected areas of the scalp of a patient, and these pulses can be spatially related to the respective patient's individual cortical anatomy, thanks to an integrated neuronavigation unit. ${ }^{45,54}$ The pulses develop into an electric field that is then capable of stimulating nervous tissue of the brain and can result in transient language disturbances of different kinds when language-related structures are affected by the induced electric field. ${ }^{18,34,63}$ Because this approach primarily allows one to parcellate the stimulated cortex into language-positive and language-negative sites, nTMS language mapping has been combined with diffusion tensor imaging fiber tracking (DTI FT) to allow the neurosurgeon to also gain information about subcortical language-related structures. $38,52,55$

The novel approach of nTMS language mapping and nTMS-based DTI FT is increasingly used in specialized neurooncological centers, and standardized mapping protocols and clinical workflow have been established..$^{30,54}$ However, the distinct impact of nTMS language mapping on clinical treatment and outcome has only been analyzed in 1 previous study, which did not routinely apply nTMSbased DTI FT in addition to cortical mapping. ${ }^{53}$ This earlier study investigated different types of brain tumors but showed that postoperative language deficits were significantly less frequent in patients who underwent preoperative nTMS language mapping when compared to a control group without preoperative nTMS language mapping, and a trend toward fewer unexpected tumor residuals was observed..$^{53}$

Against this background, this study presents the first detailed analysis of using preoperative functional data derived from both nTMS language mapping and nTMSbased DTI FT as an adjunct to awake surgery. The study evaluates the impact of this approach on functional and neurooncological outcome measures among patients suffering from highly language-eloquent gliomas.

\section{Methods}

\section{Patients and Enrollment}

Written informed consent was obtained from all patients. The study protocol was approved by the local ethics committee and was followed in accordance with the Declaration of Helsinki.

All enrolled patients were treated at our neurosurgical department between January 2011 and October 2017.
Only patients who were diagnosed with a left-hemispheric perisylvian brain tumor (according to preoperative imaging) that was postoperatively confirmed to be a low-grade glioma (LGG) or high-grade glioma (HGG) according to histopathological examination were included for this post hoc analysis of our prospectively collected cohort data. The enrolled patients underwent preoperative nTMS language mapping (and nTMS-based DTI FT) in addition to consecutive awake surgery for tumor resection, including DES and IONM. Exclusion criteria were age less than 18 years, metallic implants (e.g., cochlear implant, cardiac pacemaker, or deep brain stimulation electrodes) as contraindications for nTMS language mapping, and preoperative aphasia to a degree that would not allow preoperative or intraoperative language testing.

\section{Clinical Examinations}

Prior to surgery, each patient underwent standardized clinical examinations, which covered testing of sensory function, coordination, muscle strength, and cranial nerve function. Furthermore, the patient's Karnofsky Performance Scale (KPS) score was assessed. Language ability was evaluated by neuropsychologists using the Aachen Aphasia Test, which was supplemented by further definitions of aphasia grades: ${ }^{23,24,29,31,53} 1$ ) no deficit (grade 0); 2) mild deficit (grade 1; normal speech comprehension and/ or conversational speech with slight amnesic aphasia, adequate communication ability); 3) medium deficit (grade 2 ; minor disruption of speech comprehension and/or conversational speech, adequate communication ability); and 4) severe deficit (grade 3; major disruption of speech comprehension and/or conversational speech, clear impairment of communication ability).

The clinical examinations were repeated postoperatively during the inpatient stay and during follow-up examinations in the outpatient clinic.

\section{Magnetic Resonance Imaging}

Imaging was performed on a 3-T MRI scanner (Achieva, Philips Medical Systems, or Verio, Siemens Healthcare). Our standard minimum preoperative and follow-up protocol in patients harboring brain tumors includes a FLAIR sequence, a 3D T1-weighted gradient echo sequence with and without application of a contrast agent (gadopentetate dimeglumine; Magnograf, Marotrast $\mathrm{GmbH}$ ), and DTI sequences with 6,15 , or 32 orthogonal diffusion directions. ${ }^{54}$ Immediate postoperative imaging was performed within the first 48 hours subsequent to surgery, again using FLAIR and T1-weighted sequences as well as diffusion-weighted and T2*-weighted imaging to search for ischemic events or intracranial bleeding.

\section{Preoperative nTMS \\ nTMS Language Mapping}

Language mapping was performed prior to surgery by nTMS (eXimia NBS system; version 3.2.2 or 4.3, Nexstim Plc.). The 3D T1-weighted sequence of the respective patient was uploaded to the nTMS system and used during stimulation for neuronavigation purposes (Fig. 1). ${ }^{42,45,54,63}$ After the resting motor threshold (rMT) was determined, 


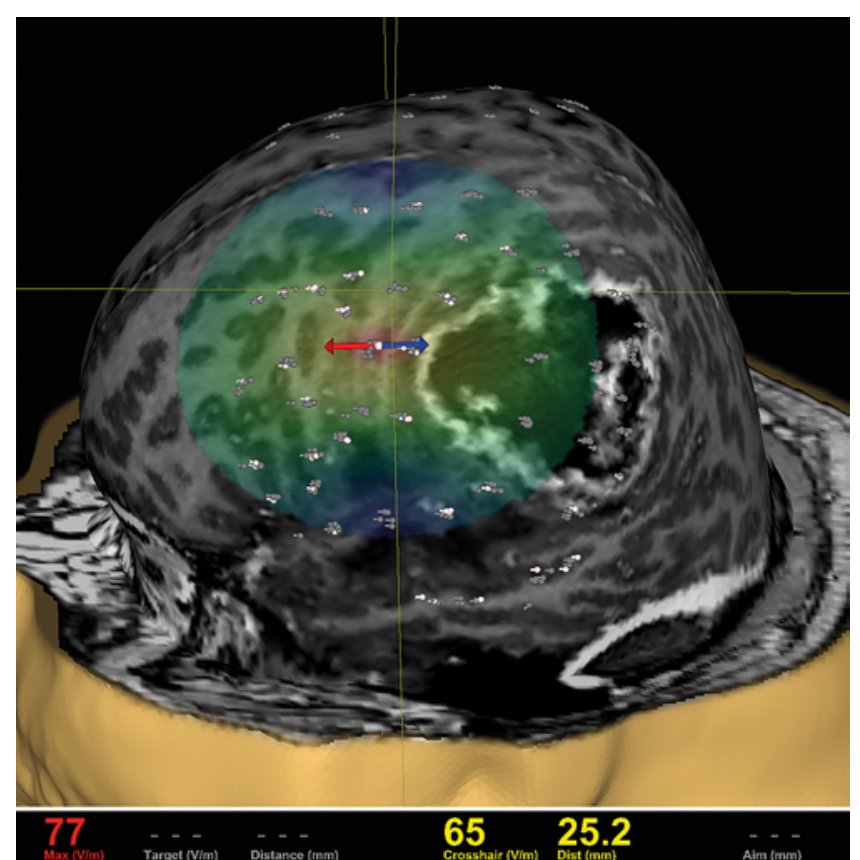

FIG. 1. Preoperative nTMS. This figure depicts nTMS language mapping with a 3D T1-weighted MRI sequence for neuronavigation purposes. Both language-negative nTMS sites (gray) and language-positive nTMS sites (white) are displayed. The colored arrow indicates the currently selected stimulation point and visualizes the localization and angulation of the induced electric field as chosen during pulse application. Only language-positive nTMS sites (white) were exported and used for subsequent nTMS-based DTI FT and during awake surgery. The figure is derived from nTMS language mapping in a patient harboring a large left-hemispheric HGG affecting the supramarginal and angular gyrus.

language mapping was performed with $80 \%-120 \%$ of the individual $\mathrm{rMT}$ and 5-7 $\mathrm{Hz}$ according to a standardized protocol using an object-naming task. ${ }^{30,32,42,57}$

Subsequent to nTMS language mapping, the video and audio data were analyzed with the aim of detecting naming errors of the following categories: no responses, performance errors, neologisms, phonological paraphasias, and semantic paraphasias. ${ }^{30,32,34,42,57}$ Hesitation errors were not routinely taken into account. Stimulation sites that showed naming errors of the above-mentioned categories were defined as language-positive, whereas all other stimulation sites were classified as language-negative.

\section{nTMS-Based DTI FT}

All language-positive sites were transferred to an external server for deterministic nTMS-based DTI FT (BrainLAB iPlan Net server, version 3.0.1; BrainLAB AG). The group of language-positive sites was first fused with the preoperatively acquired MRI sequences. Then, these sites were defined as a region of interest, and tractography was conducted with a minimum fiber length of $100 \mathrm{~mm}$ and a predefined fractional anisotropy of 0.1 or 0.15 , enabling visualization of language-related subcortical tracts. ${ }^{38,52,54,55}$ The resulting data set-consisting of preoperative MRI sequences, language-positive sites, and nTMS-based tractography-was then saved for preopera- tive surgical planning and for intraoperative application during tumor removal.

\section{Awake Surgery}

All patients underwent awake surgery for tumor removal within the scope of an asleep-awake-asleep approach using DES and IONM as previously published. ${ }^{19,37,61,62}$ The saved data set, including nTMS results of the respective patient, was available on intraoperative navigational screens during the whole procedure (BrainLAB Curve, BrainLAB AG; Fig. 2)..$^{54}$

Bupivacaine was applied for anesthesia of the galea and dura, and sedation was achieved by infusion of remifentanil and propofol. The sedation was stopped approximately 20 minutes prior to DES, which was applied during performance of an object-naming, verb-generation, and action-naming task, with the task performance evaluated by a neuropsychologist. ${ }^{29}$ DES used both bipolar (cortical stimulation) and monopolar (cortical and subcortical stimulation) electrodes (Inomed Medizintechnik GmbH) and was guided by the results of the preoperative data provided by nTMS and nTMS-based DTI FT (Fig. 2). A surface electroencephalogram was recorded to detect intraoperative seizures. Cortical sites adjacent to the ongoing resection were stimulated at least 3 times ${ }^{26,27}$ Criteria for limiting the extent of resection (EOR) during surgery were strictly based on functional borders rather than on anatomical imaging to achieve the most optimal EOR.

\section{Analysis of Clinical Outcome}

The following parameters were assessed to evaluate the clinical outcome.

\section{Craniotomy Size}

The craniotomy size was measured in anterior-posterior (AP) and lateral directions based on postoperative MRI. The overall size was then calculated.

\section{Duration of Surgery and Inpatient Stay}

The data on duration of the surgical procedure and inpatient stay were extracted from the patient charts.

\section{Extent of Resection}

The EOR was assessed macroscopically by the surgical team intraoperatively and by postoperative evaluation. Postoperative evaluation included postoperative and follow-up MRI and PET, if available. We differentiated between gross-total resection (GTR; no residual tumor present macroscopically at the end of surgery, in MRI, and in PET) and subtotal resection (STR). Any tumor residual that was confirmed by postoperative evaluation but was not expected by the neurosurgeons was defined as "unexpected residual."

\section{Language Deficit}

Language function was assessed preoperatively, postoperatively (on postoperative day 5), and during follow-up examinations (with the first regular follow-up 3 months after surgery) according to the above-mentioned grades (see Clinical Examinations above). Transient aphasia was 


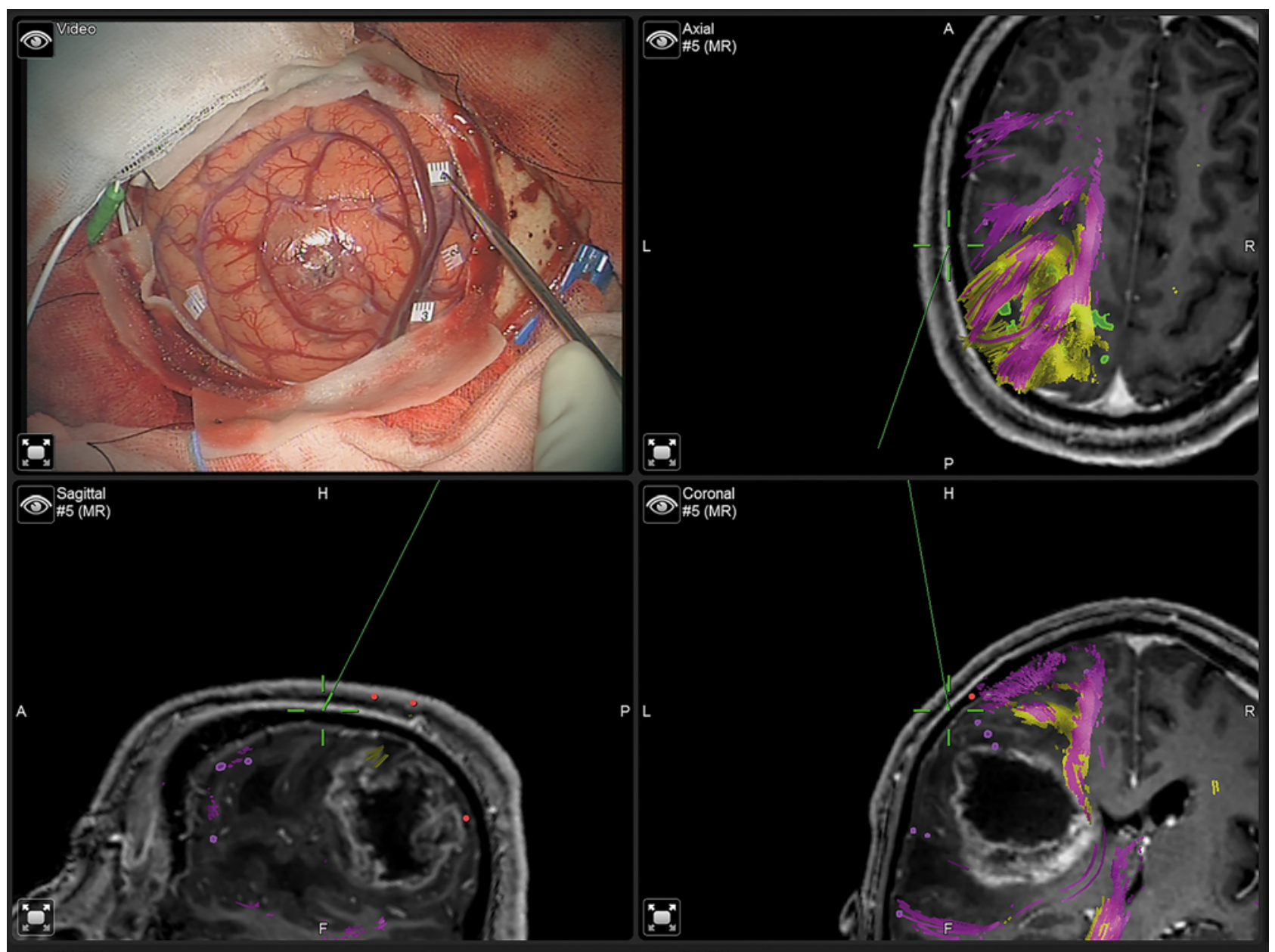

FIG. 2. Neuronavigation during awake surgery. This figure illustrates the integration of language mapping data by nTMS including nTMS-based DTI FT into neuronavigation during surgery. MRI fused with language-positive nTMS sites (purple) and nTMS-based DTI FT of language-related pathways (purple) was available on intraoperative navigational screens during the entire surgical procedure. In addition to data derived from nTMS language mapping and tractography, motor-positive nTMS sites (green) and nTMSbased DTI FT of the corticospinal tract (yellow) are shown in this illustrative case of a patient suffering from a left-hemispheric HGG affecting the supramarginal and angular gyrus.

defined as any new or worsened deficit due to surgery that resolved within the regular follow-up interval. Permanent aphasia was defined as any new or aggravated deficit due to surgery that did not resolve to the preoperative status within the regular follow-up interval.

\section{KPS Score}

The KPS score was determined preoperatively, postoperatively (on postoperative day 5), and during follow-up examinations (with the first regular follow-up 3 months after surgery). The preoperative scores were then compared against the scores registered during these later examinations.

\section{Statistical Analysis}

The parameters assessed in this study are presented as means \pm standard deviations, medians, ranges, or absolute/relative frequencies. Results are shown either for all patients together or separately for patients suffering from LGG or HGG. Values were calculated using GraphPad
Prism, which was also used for the generation of graphs (version 7.0, GraphPad Software Inc.).

\section{Results}

\section{Study Population}

Overall, 60 patients suffering from left-hemispheric LGG or HGG fulfilled the inclusion criteria and were enrolled in the present study. All included patients underwent preoperative nTMS language mapping followed by awake surgery at our neurosurgical department. Forty-five patients $(75 \%)$ also underwent preoperative nTMS-based DTI FT (nTMS-based tractography was not routinely performed in patients during the years 2011-2013). Table 1 shows relevant patient-related characteristics.

\section{Craniotomy Size}

The AP extent of the craniotomy was $7.5 \pm 1.4 \mathrm{~cm}$ (range 4.1-10.4 cm). Furthermore, the lateral extent measured $5.0 \pm 2.1 \mathrm{~cm}$ (range 1.5-8.9 cm). The resulting over- 
TABLE 1. Patient-related characteristics

\begin{tabular}{cc}
\hline \multicolumn{1}{c}{ Variable } & Value \\
\hline Age (yrs) & \\
\hline Mean \pm SD & $47.6 \pm 13.3$ \\
\hline Range & $23.9-73.9$ \\
\hline Sex (\%) & 63.3 \\
\hline Male & 36.7 \\
\hline Female & 1.7 \\
\hline WHO tumor grade $\left(\%\right.$ of patients) ${ }^{*}$ & 21.7 \\
\hline I & 30.0 \\
\hline II & 46.6 \\
\hline III & $3.7 \pm 1.4$ \\
\hline IV & $1.4-7.4$ \\
\hline Max tumor diameter (cm) $\dagger$ & \\
\hline Mean \pm SD & $21.3 \pm 16.0$ \\
\hline Range & $3.0-63.6$ \\
\hline Time to follow-up (mos)
\end{tabular}

Overall, 60 patients were enrolled, who suffered from either LGG $(n=14$ patients) or HGG ( $n=46$ patients).

* According to postoperative histopathological evaluation.

$\dagger$ According to measurements during preoperative imaging.

all area of the craniotomy accounted for $37.6 \pm 17.6 \mathrm{~cm}^{2}$ (range 10.1-80.1 $\mathrm{cm}^{2}$ ).

\section{Extent of Resection}

According to intraoperative macroscopic evaluation of the EOR by the surgical team, tumor residuals were present in 11 patients (18.3\%; LGG, 5 patients, $35.7 \%$; HGG, 6 patients, $13.0 \%$ ) at the end of the surgical procedure. According to postoperative evaluation including evaluation of imaging, 17 patients (28.3\%; LGG, 6 patients, $42.9 \%$; HGG, 11 patients, 23.9\%) showed tumor residuals. Unexpected tumor residuals were found in 6 patients $(10.0 \%$; LGG, 1 patient, 7.1\%; HGG, 5 patients, $10.9 \%$ ). Figure 3 shows the fractions of GTR or STR according to postoperative evaluation.

\section{Language Deficits}

Transient language deficits were found in 19 patients (31.7\%; LGG, 7 patients, 50.0\%; HGG, 12 patients, 26.1\%). Furthermore, 5 patients $(8.3 \%$; LGG, 1 patient, $7.1 \%$; HGG, 4 patients, $8.7 \%$ ) suffered from a new surgery-related permanent worsening of language function. However, none of the patients with surgery-related permanent deficits presented with an aphasia grade 3 at the first regular follow-up. Table 2 and Fig. 4 provide a detailed overview of the language status at different time points.

\section{Karnofsky Performance Scale Score}

The median preoperative KPS score was 90 (range 60100; LGG: median 95, range 80-100; HGG: median 90, range 60-100), and the same overall median KPS score

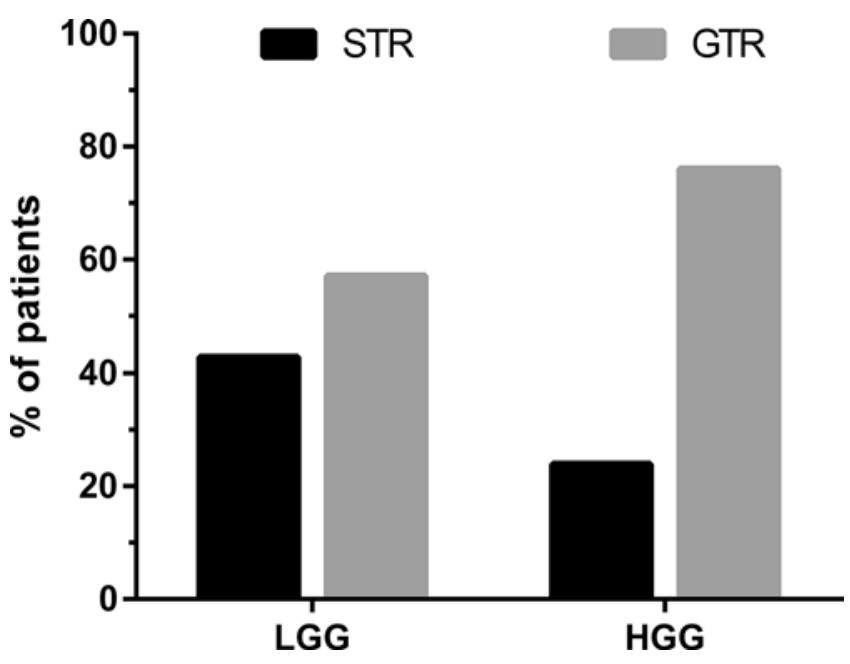

FIG. 3. Extent of resection. This bar chart shows the percentage of patients in whom GTR or STR was achieved according to postoperative evaluation, including evaluation of imaging. The fractions were calculated in relation to the total number of patients enrolled suffering from either an LGG ( $n=14$ patients) or HGG ( $n=46$ patients).

was also recorded in terms of the postoperative (range 50-100; LGG: median 90, range 70-100; HGG: median 85, range 50-100) and follow-up states (range 50-100; LGG: median 90, range 80-100; HGG: median 90, range

TABLE 2. Language deficits

\begin{tabular}{|c|c|c|}
\hline \multirow[b]{2}{*}{ Deficit } & \multicolumn{2}{|c|}{$\%$ of Patients } \\
\hline & LGG & HGG \\
\hline \multicolumn{3}{|l|}{ Preop } \\
\hline Grade 0 & 85.7 & 41.3 \\
\hline Grade 1 & 14.3 & 23.9 \\
\hline Grade 2 & 0.0 & 32.6 \\
\hline Grade 3 & 0.0 & 2.2 \\
\hline \multicolumn{3}{|l|}{ Postop } \\
\hline Grade 0 & 42.9 & 26.1 \\
\hline Grade 1 & 35.7 & 32.6 \\
\hline Grade 2 & 21.4 & 28.3 \\
\hline Grade 3 & 0.0 & 13.0 \\
\hline \multicolumn{3}{|l|}{ Follow-up } \\
\hline Grade 0 & 78.6 & 47.8 \\
\hline Grade 1 & 21.4 & 26.1 \\
\hline Grade 2 & 0.0 & 23.9 \\
\hline Grade 3 & 0.0 & 2.2 \\
\hline \multicolumn{3}{|l|}{ Surgery-related } \\
\hline Transient & 50.0 & 26.1 \\
\hline Permanent & 7.1 & 8.7 \\
\hline
\end{tabular}

This table shows the relative frequencies of patients who suffered from different grades of language deficits (grade $0=$ no deficit, grade $1=$ mild deficit, grade 2 = medium deficit, grade 3 = severe deficit) according to preoperative, postoperative (on postoperative day 5), and follow-up examinations, with the first regular follow-up occurring 3 months after surgery. Furthermore, data on surgery-related transient or permanent language deficits are provided. 


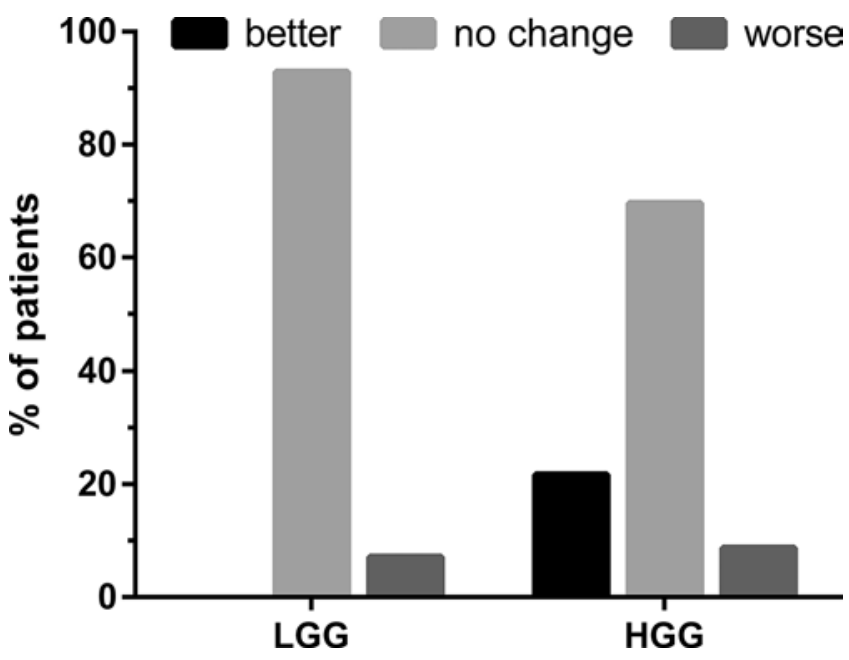

FIG. 4. Language deficits. This bar chart illustrates the percentage of patients who showed improved, unchanged, or worse language performance when comparing the preoperative to the follow-up results of clinical examination. The fractions were calculated in relation to the total number of patients enrolled suffering from either an LGG $(n=14$ patients) or HGG ( $n=46$ patients).

50-100). The median difference in KPS scores when comparing the preoperative to the follow-up state was 0 (range -30 to 20 ; LGG: median 0, range -20 to 10 , HGG: median 0 , range -30 to 20 ).

\section{Duration of Surgery and Inpatient Stay}

For all enrolled patients combined, the duration of the entire awake procedure from the skin incision to the suture at the end of surgery was $256.5 \pm 52.7$ minutes (range 156.0-405.0 minutes). The median inpatient stay was 9.0 days (range 4.0-34.0 days).

\section{Discussion}

\section{Preoperative Language Mapping}

This study presented the combination of preoperative nTMS language mapping with nTMS-based DTI FT and awake surgery for the resection of highly languageeloquent brain tumors as a modern setup. It furthermore analyzed a variety of clinical outcome parameters of this novel approach, which has not yet been undertaken for this combination.

The need for individual language mapping is based on the high potential of plastic reorganization of cortical and subcortical language-related structures as repeatedly reported for patients suffering from brain tumors. $3,7,8,14,17,59$ Hence, patients with tumor-induced plastic reshaping are unlikely to present highly language-eloquent structures only at sites known from the textbook; they rather present with at least partial reallocation of sites within the tumor-affected or contralateral hemisphere..$^{3,7,8,14,17,59}$ This reallocation can be mapped by DES during awake surgery, but further techniques are commonly used preoperatively to assess plastic reshaping noninvasively and the spatial relationship between language-related structures and the tumor volume. This can help during resection planning and intraoperative guidance. Among these techniques,
fMRI and MEG are most frequently applied. However, both have been shown to harbor relevant limitations when considering mapping of tumor-affected brains. Concerning fMRI, it has been demonstrated that the blood oxygenation level-dependent (BOLD) contrast lacks accuracy in patients with changed intracranial oxygen levels, which are regularly present in the vicinity of gliomas due to their glucose and oxygen consumption from surrounding brain tissue. ${ }^{13,15,22,51}$ In contrast to BOLD fMRI, preoperative $\mathrm{MEG}$ in patients harboring brain tumors is not reported to severely suffer from altered oxygen levels. However, MEG represents a more expensive alternative that is not widely available. Furthermore, preoperative MEG has primarily been applied to assess language lateralization without distinct detection of specific language-related sites because it identifies regions of cortical activity without testing of single areas, as DES does. ${ }^{12,28,41,63}$ Another limitation is related to analysis algorithms: a dipole analysis is commonly used, which is known to be sensitive to noise and the selection of model parameters. ${ }^{12}$ More robust methods are currently developed for preoperative MEG applications; however, the goal of any analysis mostly remains to determine language lateralization without gaining explicit data about single language-related sites..$^{20,21}$

Due to the shortcomings reported for fMRI and MEG, nTMS has been recently introduced as an alternative for language mapping in patients harboring perisylvian brain tumors. ${ }^{42,56,63}$ The approach uses noninvasive but targeted stimulation of the brain with the aim of eliciting language disturbances during performance of a task; thus, the technique is principally comparable to DES during awake surgery, which reflects the current gold standard in functional brain mapping. ${ }^{60-62}$ The noninvasive nature of nTMS enables its preoperative application as a neurosurgical tool for resection planning and guidance, which has been shown to be safe and well tolerated. ${ }^{64}$ Moreover, preoperative nTMS language mapping has already been compared to preoperative fMRI and MEG in combination with DES, with the result that nTMS correlates well with DES for neurosurgical applications. ${ }^{26,56,63}$ In this context, preoperative nTMS detected language-related cortical sites in the vicinity of an HGG that correlated well with DES, whereas fMRI failed to demonstrate the same sites and wrongly indicated language dominance within the unaffected hemisphere. ${ }^{56}$ Furthermore, nTMS language maps were shown to correlate well with those generated by DES, while MEG lacked the same level of correlation in a cohort consisting of 12 patients suffering from language-eloquent tumors. ${ }^{63}$

The addition of nTMS-based DTI FT to nTMS language mapping, as presented in this study, enables the visualization of subcortical language-related pathways. Although the results of nTMS-based DTI FT of language-related pathways have not yet been confirmed by subcortical DES due to the novelty of the approach, this method appears to be helpful in guiding resection trajectories and resection per se. ${ }^{25}$ In the present study, $28.3 \%$ of patients initially harboring highly language-eloquent gliomas showed residuals according to postoperative evaluation. Preoperative nTMS language mapping combined with nTMS-based DTI FT, incorporated into intraoperative neuronavigation, may lead to an increased confidence of the neurosurgeon 
in identifying the individual functional anatomy of the patient, thus possibly leading to more radical resections. Similar associations have already been suggested in terms of intraoperative techniques such as DES and IONM. ${ }^{5,9,60}$

Concerning surgery-related language deterioration, $31.7 \%$ of patients enrolled in this study suffered from transient surgery-related language deficits, whereas $8.3 \%$ of patients suffered from permanent deficits; however, no patient suffered from a severe surgery-related permanent deficit. In this context, various prior studies examined short- and long-term outcome following DES in LGG and HGG. Immediate transient postoperative deterioration of language function has been shown to exist in 14\%-50\% of patients. ${ }^{11,49,66}$ Permanent new surgery-related decline of function can be observed in approximately $5 \%-20 \%$ of patients on average. . $^{1,450,65}$

Of note, the selection of patients for awake surgery can differ significantly between centers and likewise plays a highly relevant role regarding clinical outcome. ${ }^{58}$ During the time of enrollment for the present study, no patient was determined to harbor a nonresectable tumor, thus leading to the inclusion of a consecutive series of patients with highly language-eloquent lesions. Inclusion of such patients with potentially high risks of surgery-related permanent deficits due to tumor location can be obtained by detailed preoperative information as outlined in this report, which also helps to qualify more patients for a surgical approach. Thus, such valuable noninvasive data might lead not only to a considerably low rate of deficits but also to a higher number of patients undergoing surgery.

\section{Limitations of the Study}

Although this is the first study to analyze various outcome parameters of the combined approach of nTMS language mapping followed by nTMS-based DTI FT and awake surgery, we have to acknowledge some limitations. First, the present study analyzed clinical outcome without a control group; thus, follow-up studies that include randomized controlled trials are needed to prove the optimized outcome in comparison to patients who do not undergo such an extensive preoperative workup. Moreover, the lack of a control group-with random assignment of patients to the nTMS group or control group and the investigators blinded to these assignments - can principally result in a bias regarding the evaluation of clinical outcome parameters, with trends toward favorable evaluations. The outcome data shown in the present study were primarily collected within the scope of clinical routine and, thus, reflect the state of the patients that was documented by experienced neurosurgeons and neuroradiologists. Furthermore, we used comparatively broad and standardized categories for evaluation (e.g., 4 grades of aphasia, KPS scores), which are well defined and should allow for clear allocation of patients without much margin for misinterpretation. Second, a multimodal study comparing preoperative nTMS language mapping and tractography to fMRI or MEG prior to awake surgery seems reasonable to evaluate whether this novel approach is favorable; this is also not provided by this study. As aforementioned, modality comparisons have already been performed, but these focused on accuracy of preoperative methods in comparison to DES without distinct analyses of clinical outcome. ${ }^{26,56,63}$ Third, nTMS language mapping has been shown to correlate well with DES; nevertheless, it suffers from a comparatively low specificity and positive predictive value, combined with a high overall sensitivity and a high negative predictive value. ${ }^{33}$ The high sensitivity may indicate that not all language-positive nTMS sites are essential or nonresectable sites from a neurosurgical point of view but are rather involved in language processing. ${ }^{33}$ Thus, negative nTMS language mapping was shown to correlate well with negative DES, which is actually sufficient for neurosurgical clinical application, because even for awake surgery, some centers rely fully on mapped but language-negative cortical areas. ${ }^{19,63}$ Nevertheless, further nTMS protocol optimizations may help to increase the positive correlation to DES. One previous study already showed the successful stand-alone use of nTMS and nTMS-based DTI FT in very preselected patients who were unable to undergo awake surgery. ${ }^{25}$ Yet, if awake surgery is possible, this should always be the first choice.

\section{Conclusions}

The present study combined nTMS language mapping with nTMS-based DTI FT to provide a comprehensive preoperative approach for anatomico-functional language assessment among patients suffering from highly language-eloquent gliomas. It reflects the first study to analyze various clinical outcome parameters in the context of this novel approach. Our preoperative setup, combined with subsequent awake surgery including DES and IONM, resulted in good clinical outcome.

\section{References}

1. Brell M, Ibáñez J, Caral L, Ferrer E: Factors influencing surgical complications of intra-axial brain tumours. Acta Neurochir (Wien) 142:739-750, 2000

2. Brown TJ, Brennan MC, Li M, Church EW, Brandmeir NJ, Rakszawski KL, et al: Association of the extent of resection with survival in glioblastoma: a systematic review and metaanalysis. JAMA Oncol 2:1460-1469, 2016

3. Chang EF, Breshears JD, Raygor KP, Lau D, Molinaro AM, Berger MS: Stereotactic probability and variability of speech arrest and anomia sites during stimulation mapping of the language dominant hemisphere. J Neurosurg 126:114-121, 2017

4. Chang SM, Parney IF, McDermott M, Barker FG II, Schmidt $\mathrm{MH}$, Huang W, et al: Perioperative complications and neurological outcomes of first and second craniotomies among patients enrolled in the Glioma Outcome Project. J Neurosurg 98:1175-1181, 2003

5. De Witt Hamer PC, Robles SG, Zwinderman AH, Duffau H, Berger MS: Impact of intraoperative stimulation brain mapping on glioma surgery outcome: a meta-analysis. J Clin Oncol 30:2559-2565, 2012

6. Duffau H: Is non-awake surgery for supratentorial adult low-grade glioma treatment still feasible? Neurosurg Rev 41:133-139, 2018

7. Duffau H: Lessons from brain mapping in surgery for lowgrade glioma: insights into associations between tumour and brain plasticity. Lancet Neurol 4:476-486, 2005

8. Duffau H: Stimulation mapping of white matter tracts to study brain functional connectivity. Nat Rev Neurol 11:255265,2015 
9. Duffau H, Lopes M, Arthuis F, Bitar A, Sichez JP, Van Effenterre R, et al: Contribution of intraoperative electrical stimulations in surgery of low grade gliomas: a comparative study between two series without (1985-96) and with (1996-2003) functional mapping in the same institution. J Neurol Neurosurg Psychiatry 76:845-851, 2005

10. Duffau H, Mandonnet E: The "onco-functional balance" in surgery for diffuse low-grade glioma: integrating the extent of resection with quality of life. Acta Neurochir (Wien) 155:951-957, 2013

11. Duffau H, Moritz-Gasser S, Gatignol P: Functional outcome after language mapping for insular World Health Organization Grade II gliomas in the dominant hemisphere: experience with 24 patients. Neurosurg Focus 27(2):E7, 2009

12. Findlay AM, Ambrose JB, Cahn-Weiner DA, Houde JF, Honma S, Hinkley LB, et al: Dynamics of hemispheric dominance for language assessed by magnetoencephalographic imaging. Ann Neurol 71:668-686, 2012

13. Fraga de Abreu VH, Peck KK, Petrovich-Brennan NM, Woo KM, Holodny AI: Brain tumors: the influence of tumor type and routine MR imaging characteristics at BOLD functional MR imaging in the primary motor gyrus. Radiology 281:876-883, 2016

14. Ghinda CD, Duffau H: Network plasticity and intraoperative mapping for personalized multimodal management of diffuse low-grade gliomas. Front Surg 4:3, 2017

15. Giussani C, Roux FE, Ojemann J, Sganzerla EP, Pirillo D, Papagno C: Is preoperative functional magnetic resonance imaging reliable for language areas mapping in brain tumor surgery? Review of language functional magnetic resonance imaging and direct cortical stimulation correlation studies. Neurosurgery 66:113-120, 2010

16. Håberg A, Kvistad KA, Unsgård G, Haraldseth O: Preoperative blood oxygen level-dependent functional magnetic resonance imaging in patients with primary brain tumors: clinical application and outcome. Neurosurgery 54:902-915, 2004

17. Herbet G, Maheu M, Costi E, Lafargue G, Duffau H: Mapping neuroplastic potential in brain-damaged patients. Brain 139:829-844, 2016

18. Hernandez-Pavon JC, Mäkelä N, Lehtinen H, Lioumis $P$, Mäkelä JP: Effects of navigated TMS on object and action naming. Front Hum Neurosci 8:660, 2014

19. Hervey-Jumper SL, Li J, Lau D, Molinaro AM, Perry DW, Meng L, et al: Awake craniotomy to maximize glioma resection: methods and technical nuances over a 27 -year period. J Neurosurg 123:325-339, 2015

20. Hirata M, Goto T, Barnes G, Umekawa Y, Yanagisawa T, Kato A, et al: Language dominance and mapping based on neuromagnetic oscillatory changes: comparison with invasive procedures. J Neurosurg 112:528-538, 2010

21. Hirata M, Kato A, Taniguchi M, Saitoh Y, Ninomiya H, Ihara A, et al: Determination of language dominance with synthetic aperture magnetometry: comparison with the Wada test. Neuroimage 23:46-53, 2004

22. Holodny AI, Schulder M, Liu WC, Wolko J, Maldjian JA, Kalnin AJ: The effect of brain tumors on BOLD functional MR imaging activation in the adjacent motor cortex: implications for image-guided neurosurgery. AJNR Am J Neuroradiol 21:1415-1422, 2000

23. Huber W, Poeck K, Willmes K: The Aachen Aphasia Test. Adv Neurol 42:291-303, 1984

24. Ille S, Kulchytska N, Sollmann N, Wittig R, Beurskens E, Butenschoen VM, et al: Hemispheric language dominance measured by repetitive navigated transcranial magnetic stimulation and postoperative course of language function in brain tumor patients. Neuropsychologia 91:50-60, 2016

25. Ille S, Sollmann N, Butenschoen VM, Meyer B, Ringel F, Krieg SM: Resection of highly language-eloquent brain lesions based purely on rTMS language mapping without awake surgery. Acta Neurochir (Wien) 158:2265-2275, 2016

26. Ille S, Sollmann N, Hauck T, Maurer S, Tanigawa N, Obermueller T, et al: Combined noninvasive language mapping by navigated transcranial magnetic stimulation and functional MRI and its comparison with direct cortical stimulation. J Neurosurg 123:212-225, 2015

27. Ille S, Sollmann N, Hauck T, Maurer S, Tanigawa N, Obermueller T, et al: Impairment of preoperative language mapping by lesion location: a functional magnetic resonance imaging, navigated transcranial magnetic stimulation, and direct cortical stimulation study. J Neurosurg 123:314-324, 2015

28. Kamada K, Sawamura Y, Takeuchi F, Kuriki S, Kawai K, Morita A, et al: Expressive and receptive language areas determined by a non-invasive reliable method using functional magnetic resonance imaging and magnetoencephalography. Neurosurgery 60:296-306, 2007

29. Kelm A, Sollmann N, Ille S, Meyer B, Ringel F, Krieg SM: Resection of gliomas with and without neuropsychological support during awake craniotomy-effects on surgery and clinical outcome. Front Oncol 7:176, 2017

30. Krieg SM, Lioumis P, Mäkelä JP, Wilenius J, Karhu J, Hannula H, et al: Protocol for motor and language mapping by navigated TMS in patients and healthy volunteers; workshop report. Acta Neurochir (Wien) 159:1187-1195, 2017

31. Krieg SM, Sollmann N, Hauck T, Ille S, Meyer B, Ringel F: Repeated mapping of cortical language sites by preoperative navigated transcranial magnetic stimulation compared to repeated intraoperative DCS mapping in awake craniotomy. BMC Neurosci 15:20, 2014

32. Krieg SM, Sollmann N, Tanigawa N, Foerschler A, Meyer B, Ringel F: Cortical distribution of speech and language errors investigated by visual object naming and navigated transcranial magnetic stimulation. Brain Struct Funct 221:22592286, 2016

33. Krieg SM, Tarapore PE, Picht T, Tanigawa N, Houde J, Sollmann N, et al: Optimal timing of pulse onset for language mapping with navigated repetitive transcranial magnetic stimulation. Neuroimage 100:219-236, 2014

34. Lioumis P, Zhdanov A, Mäkelä N, Lehtinen H, Wilenius J, Neuvonen T, et al: A novel approach for documenting naming errors induced by navigated transcranial magnetic stimulation. J Neurosci Methods 204:349-354, 2012

35. Mäkelä JP, Forss N, Jääskeläinen J, Kirveskari E, Korvenoja A, Paetau R: Magnetoencephalography in neurosurgery. Neurosurgery 61 (1 Suppl):147-165, 2007

36. McGirt MJ, Mukherjee D, Chaichana KL, Than KD, Weingart JD, Quinones-Hinojosa A: Association of surgically acquired motor and language deficits on overall survival after resection of glioblastoma multiforme. Neurosurgery 65:463-470, 2009

37. Meng L, McDonagh DL, Berger MS, Gelb AW: Anesthesia for awake craniotomy: a how-to guide for the occasional practitioner. Can J Anaesth 64:517-529, 2017

38. Negwer C, Ille S, Hauck T, Sollmann N, Maurer S, Kirschke JS, et al: Visualization of subcortical language pathways by diffusion tensor imaging fiber tracking based on rTMS language mapping. Brain Imaging Behav 11:899-914, 2017

39. Ottenhausen M, Krieg SM, Meyer B, Ringel F: Functional preoperative and intraoperative mapping and monitoring: increasing safety and efficacy in glioma surgery. Neurosurg Focus 38(1):E3, 2015

40. Papanicolaou AC, Simos PG, Breier JI, Zouridakis G, Willmore LJ, Wheless JW, et al: Magnetoencephalographic mapping of the language-specific cortex. J Neurosurg 90:85-93, 1999

41. Papanicolaou AC, Simos PG, Castillo EM, Breier JI, Sarkari S, Pataraia E, et al: Magnetocephalography: a noninvasive al- 
ternative to the Wada procedure. J Neurosurg 100:867-876, 2004

42. Picht T, Krieg SM, Sollmann N, Rösler J, Niraula B, Neuvonen T, et al: A comparison of language mapping by preoperative navigated transcranial magnetic stimulation and direct cortical stimulation during awake surgery. Neurosurgery 72:808-819, 2013

43. Roessler K, Donat M, Lanzenberger R, Novak K, Geissler A, Gartus A, et al: Evaluation of preoperative high magnetic field motor functional MRI (3 Tesla) in glioma patients by navigated electrocortical stimulation and postoperative outcome. J Neurol Neurosurg Psychiatry 76:1152-1157, 2005

44. Roux FE, Boulanouar K, Lotterie JA, Mejdoubi M, LeSage JP, Berry I: Language functional magnetic resonance imaging in preoperative assessment of language areas: correlation with direct cortical stimulation. Neurosurgery 52:13351347,2003

45. Ruohonen J, Karhu J: Navigated transcranial magnetic stimulation. Neurophysiol Clin 40:7-17, 2010

46. Sacko O, Lauwers-Cances V, Brauge D, Sesay M, Brenner A, Roux FE: Awake craniotomy vs surgery under general anesthesia for resection of supratentorial lesions. Neurosurgery 68:1192-1199, 2011

47. Sanai N, Berger MS: Glioma extent of resection and its impact on patient outcome. Neurosurgery 62:753-764, 264266, 2008

48. Sanai N, Berger MS: Recent surgical management of gliomas. Adv Exp Med Biol 746:12-25, 2012

49. Sanai N, Mirzadeh Z, Berger MS: Functional outcome after language mapping for glioma resection. N Engl J Med 358:18-27, 2008

50. Sawaya R, Hammoud M, Schoppa D, Hess KR, Wu SZ, Shi WM, et al: Neurosurgical outcomes in a modern series of 400 craniotomies for treatment of parenchymal tumors. Neurosurgery 42:1044-1056, 1998

51. Schreiber A, Hubbe U, Ziyeh S, Hennig J: The influence of gliomas and nonglial space-occupying lesions on bloodoxygen-level-dependent contrast enhancement. AJNR Am J Neuroradiol 21:1055-1063, 2000

52. Sollmann N, Giglhuber K, Tussis L, Meyer B, Ringel F, Krieg SM: nTMS-based DTI fiber tracking for language pathways correlates with language function and aphasia - a case report. Clin Neurol Neurosurg 136:25-28, 2015

53. Sollmann N, Ille S, Hauck T, Maurer S, Negwer C, Zimmer $\mathrm{C}$, et al: The impact of preoperative language mapping by repetitive navigated transcranial magnetic stimulation on the clinical course of brain tumor patients. BMC Cancer 15:261, 2015

54. Sollmann N, Meyer B, Krieg SM: Implementing functional preoperative mapping in the clinical routine of a neurosurgical department: technical note. World Neurosurg 103:94105,2017

55. Sollmann N, Negwer C, Ille S, Maurer S, Hauck T, Kirschke JS, et al: Feasibility of nTMS-based DTI fiber tracking of language pathways in neurosurgical patients using a fractional anisotropy threshold. J Neurosci Methods 267:45-54, 2016

56. Sollmann N, Picht T, Mäkelä JP, Meyer B, Ringel F, Krieg SM: Navigated transcranial magnetic stimulation for preoperative language mapping in a patient with a left frontoopercular glioblastoma. J Neurosurg 118:175-179, 2013

57. Sollmann N, Tanigawa N, Ringel F, Zimmer C, Meyer B, Krieg SM: Language and its right-hemispheric distribution in healthy brains: an investigation by repetitive transcranial magnetic stimulation. Neuroimage 102:776-788, 2014
58. Southwell DG, Birk HS, Han SJ, Li J, Sall JW, Berger MS: Resection of gliomas deemed inoperable by neurosurgeons based on preoperative imaging studies. J Neurosurg [epub ahead of print November 10, 2017; DOI: 10.3171/2017.5.JNS17166]

59. Southwell DG, Hervey-Jumper SL, Perry DW, Berger MS: Intraoperative mapping during repeat awake craniotomy reveals the functional plasticity of adult cortex. J Neurosurg 124:1460-1469, 2016

60. Szelényi A, Bello L, Duffau H, Fava E, Feigl GC, Galanda $\mathrm{M}$, et al: Intraoperative electrical stimulation in awake craniotomy: methodological aspects of current practice. Neurosurg Focus 28(2):E7, 2010

61. Talacchi A, Santini B, Casagrande F, Alessandrini F, Zoccatelli G, Squintani GM: Awake surgery between art and science. Part I: clinical and operative settings. Funct Neurol 28:205-221, 2013

62. Talacchi A, Santini B, Casartelli M, Monti A, Capasso R, Miceli G: Awake surgery between art and science. Part II: language and cognitive mapping. Funct Neurol 28:223-239, 2013

63. Tarapore PE, Findlay AM, Honma SM, Mizuiri D, Houde JF, Berger MS, et al: Language mapping with navigated repetitive TMS: proof of technique and validation. Neuroimage 82:260-272, 2013

64. Tarapore PE, Picht T, Bulubas L, Shin Y, Kulchytska N, Meyer B, et al: Safety and tolerability of navigated TMS for preoperative mapping in neurosurgical patients. Clin Neurophysiol 127:1895-1900, 2016

65. Taylor MD, Bernstein M: Awake craniotomy with brain mapping as the routine surgical approach to treating patients with supratentorial intraaxial tumors: a prospective trial of 200 cases. J Neurosurg 90:35-41, 1999

66. Wilson SM, Lam D, Babiak MC, Perry DW, Shih T, Hess CP, et al: Transient aphasias after left hemisphere resective surgery. J Neurosurg 123:581-593, 2015

\section{Disclosures}

Dr. Krieg is a consultant for BrainLAB AG and Nexstim Plc. Drs. Ringel and Meyer are consultants for BrainLAB AG.

\section{Author Contributions}

Conception and design: Krieg, Sollmann. Acquisition of data: Krieg, Sollmann, Kelm, Ille, Schröder, Ringel, Meyer. Analysis and interpretation of data: Krieg, Sollmann, Kelm, Ille, Schröder. Drafting the article: Krieg, Sollmann. Critically revising the article: Krieg. Reviewed submitted version of manuscript: Krieg, Kelm, Ille, Schröder, Zimmer, Ringel, Meyer. Approved the final version of the manuscript on behalf of all authors: Krieg. Statistical analysis: Sollmann. Administrative/technical/material support: Krieg, Zimmer, Ringel, Meyer. Study supervision: Krieg, Ringel, Meyer.

\section{Supplemental Information}

\section{Current Affiliation}

Dr. Ringel: Department of Neurosurgery, Universitätsmedizin Mainz, Germany.

\section{Correspondence}

Sandro M. Krieg: Klinikum rechts der Isar, Technische Universität München, Germany. sandro.krieg@tum.de. 